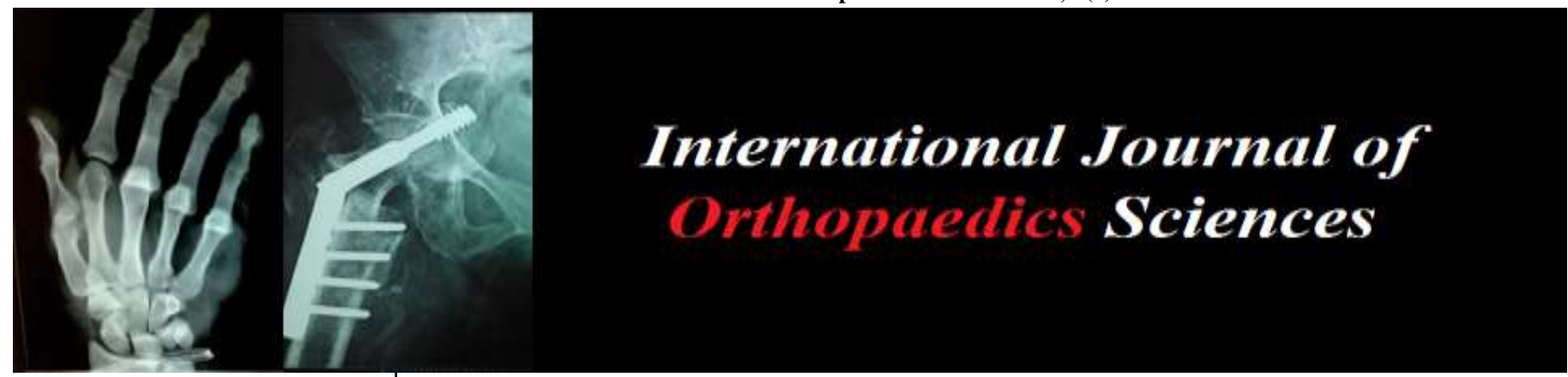

E-ISSN: 2395-1958

P-ISSN: 2706-6630

IJOS 2021; 7(1): 684-690

(C) 2021 IJOS

www.orthopaper.com

Received: 29-10-2020

Accepted: 23-12-2020

Dr. Mithun Shetty

Assistant Professor, MS

Orthopaedics, Department of Orthopaedics, AJ Institute of

Medical Sciences, Mangalore,

Karnataka, India

Dr. Shashi Kumar MS

Junior Resident, MS

Orthopaedics, Department of Orthopaedics, AJ Institute of

Medical Sciences, Mangalore,

Karnataka, India

Dr. Shree Krishnananda Sagar Junior Resident, MS

Orthopaedics, Department of Orthopaedics, AJ Institute of Medical Sciences, Mangalore,

Karnataka, India

Dr. Anusree Anil Kumar

Intern, MBBS, Department of Orthopaedics, AJ Institute of Medical Sciences, Mangalore, Karnataka, India
Corresponding Author: Dr. Shashi Kumar MS Junior Resident, MS Orthopaedics, Department of Orthopaedics, AJ Institute of Medical Sciences, Mangalore, Karnataka, India

\section{Pre fixation compression screw as a cutting-edge technique for varus correction during proximal femoral nailing for intertrochanteric fractures: A study on 46 cases}

\author{
Dr. Mithun Shetty, Dr. Shashi Kumar MS, Dr. Shree Krishnananda \\ Sagar and Dr. Anusree Anil Kumar
}

DOI: https://doi.org/10.22271/ortho.2021.v7.i1k.2560

Abstract

Background: Achieving reduction prior to fixation is a key aspect in successfully managing intertrochanteric fractures. In this study of proximal femoral nailing for intertrochanteric fractures, a novel technique of utilizing pre-fixation compression screw was introduced to achieve and maintain reduction of femoral neck shaft angle.

Materials and Methods: The current prospective study was conducted over a period of six months between July 2018 and January 2019 among patients who underwent surgical fixation by PFN for intertrochanteric fractures of femur. Pre-fixation compression screw was used in all the cases to achieve and maintain correction of varus. Pre-operative, intra-operative and post- operative neck shaft angles were documented. Data was analysed by using Statistical Package for Social Sciences (SPSS). The results were expressed as percentages and graphs.

Results: There is significant improvement in correction of varus (average increase of neck shaft angle $=14.04$ degrees) with the use of pre-fixation screw technique. This procedure did neither increase the blood loss (average blood loss $=34.67 \mathrm{ml}$ ) during the procedure nor the duration (average duration $=$ $19.46 \mathrm{~min}$ ) of the procedure.

Conclusion: Pre-fixation compression screw can be used as an effective alternative for varus correction in cases of simple intertrochanteric fractures undergoing PFN fixation with added intra-op and post-op advantages compared to other methods of reduction.

Keywords: Intertrochanteric fractures, proximal femoral nailing, pre-fixation screw, varus correction

\section{Introduction}

Intertrochanteric fractures (IT) of the femur are common occurrence in the geriatric age group [1]. Internal fixation with minimally invasive approach would be ideal to reduce morbidity at this age group ${ }^{[2,3,4]}$. Failure to achieve good reduction is one of the important reasons for implant failures and thus compelling the patient to undergo multiple salvage procedures ${ }^{[5]}$. Multiple surgical procedures would create concerns on increasing morbidities at this fragile age group and hence it is imperative to obtain good reduction prior to implantation. Correction of varus, posterior sag and rotations are important objectives mandatory to achieve prior to fixation with an appropriate implant ${ }^{[6,7]}$. Proximal femoral nailing has been considered as the implant of choice over dynamic hip screw in geriatric age group as it reduces extensive soft tissue dissection, decreases blood loss, reduces the duration of the procedure and consequently reducing the morbidity $[8,9,10]$. Correction of varus is an important pre-requisite to achieve stability in IT fractures which can be achieved by traction on the fracture table and placing the hip in neutral or slight abduction ${ }^{[11]}$. Dynamic hip screw would be an ideal implant of choice for relatively stable AO 31. A.1 type fractures where the reduction is easily achieved by hip in neutral or abduction. However, it is associated with increased morbidity due to the more invasive nature of the procedure ${ }^{[2]}$.

In proximal femoral nailing, patient must be positioned on the fracture table with traction and affected hip in adducted position to facilitate nail entry. 
An important caveat with proximal femoral nailing is maintaining valgus angle when the hip is forced into adduction. Many reduction manoeuvres have been explained in the literatures which have their own drawbacks. A common method used to maintain valgus is by using K-wires for prefixation after obtaining reduction in abducted position of the hip ${ }^{[11]}$. K-wires fails to maintain valgus in many instances when hip is brought to adduction owing to the osteoporotic nature of bone in elderly and due to powerful abductor muscle forces in a relatively young patient. Use of schanz pins and bone hook in pyriform fossa are few of the other techniques mentioned in literature for maintaining reduction ${ }^{[12]}$. However, these techniques render the surgical procedure cumbersome while implanting PFN with an external zig.

In simple intertrochanteric fractures, the fracture line tends to open up near the greater trochanter as the hip is positioned in adduction, forcing the proximal fracture fragment into varus. To prevent this eccentric distraction of fracture fragments, it is imperative to have a compressive device in the proximal half of the fracture line. This would not only provide a sturdy fixation that prevents the worsening of varus angulation when hip is positioned in adduction but also aids in obtaining valgus correction. Furthermore, considering the concerns on increasing morbidity while executing this additional step, it is essential to have a technique which is not very complex. In this study, we have made an attempt to analyse the effect of using an eccentric cannulated cancellous screw as a prefixation technique to achieve and maintain varus correction in simple intertrochanteric fractures. Once the fracture reduction was maintained in adducted position of hip, definitive fixation with PFN was done.

\section{Methodology}

\section{Study design and participants}

The current prospective study was conducted over a period of six months from July 2018 to January 2019 among patients admitted with intertrochanteric fractures (A1.1, A1.2, A1.3 and A2.1 as per A.O classification) at a tertiary care teaching hospital in South India. Patients of age more than 50 years were included in the study. Patients who had open fracture, poly-trauma, subtrochanteric fracture, reverse oblique and metastatic fractures were excluded.

\section{Study instrument}

Extensive review of literature was done to evaluate the pros and cons of various reduction methods for trochanteric fractures. This study presents a cutting-edge technique of trochanteric fracture reduction and maintaining neck shaft angle of femur with pre-fixation compression screw. The pros and cons of surgery were compared to other reduction techniques to further validate the results.

\section{Surgical technique}

All the patients involved in the study were operated by the same surgeon and under spinal anaesthesia. Patient was positioned supine on the fracture table with involved limb fixed to traction apparatus and contralateral limb placed in lithotomy position. Fracture reduction was obtained with limb in abduction or neutral position at the hip joint with counter traction provided by perineal post. Opposite limb was put in full abduction over lithotomy support to give enough space for the $\mathrm{C}$-arm in between the legs. Fluoroscopic confirmation of fracture reduction was done before the limb was scrubbed, painted and draped in an aseptic manner.

To prevent varus displacement of reduced fragment, a percutaneous guide wire was placed under fluoroscopy guidance in the anterior and superior aspect of the neck of femur. A cannulated cancellous screw was placed after drilling to provide eccentric compression. This manoeuvre prevented loss of reduction on adducting the limb or during nail insertion. It also helped in closing the fracture site which would inevitably become the nail entry site as shown in (Fig $1 \mathrm{~A}$ and $\mathrm{B}$.).
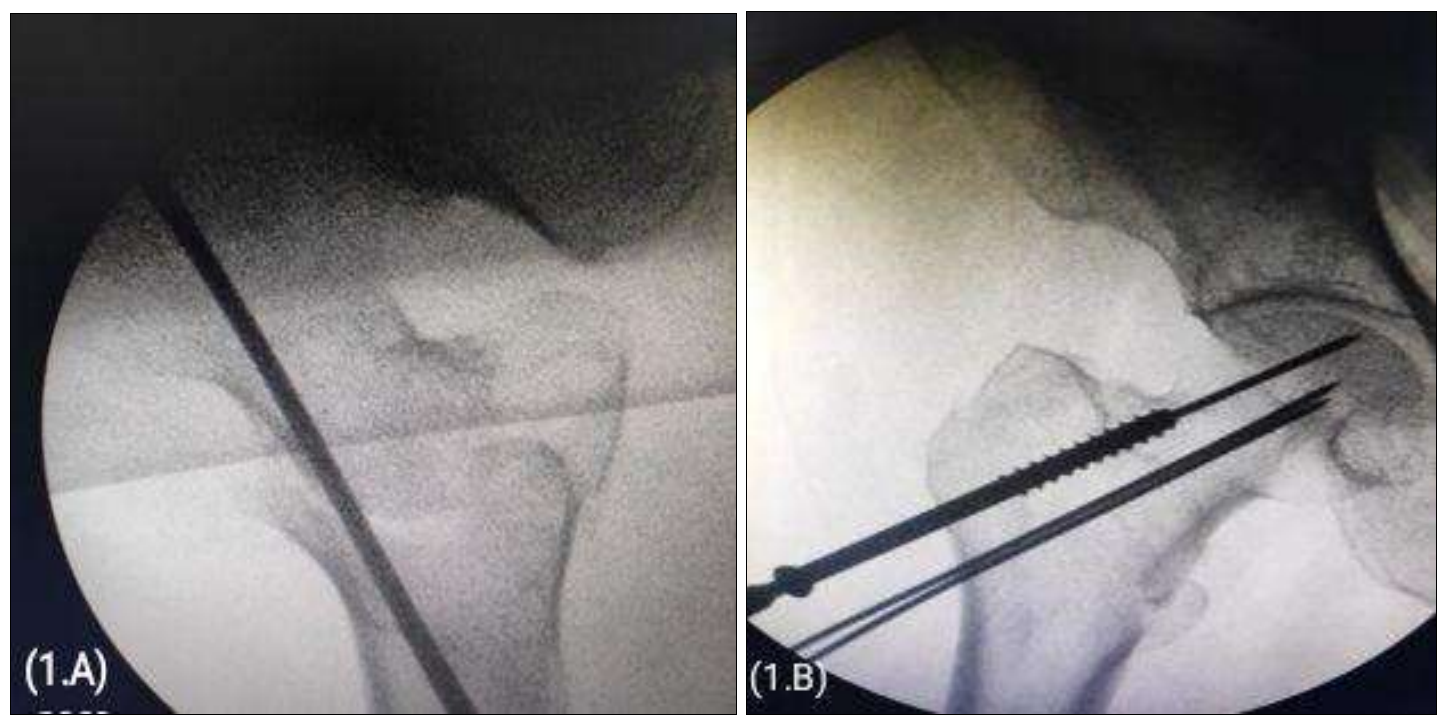

Fig 1A and B: (A) Illustrates reduced IT fracture with eccentrically placed guide wire and, (B) Pre-fixation compression screw placement to prevent displacement on adduction of limb.

A $2 \mathrm{~cm}$ incision was taken above the tip of the greater trochanter. Using an AWL, nail entry space is created at the tip of the greater trochanter. Reaming of the femur was done in relatively younger patients who had good bone quality and narrow canal. The compression screw would prevent opening up of fracture site during the insertion of nail and hence prevent inadvertent varus. The eccentrically placed cannulated cancellous screw will not pose any problem in nail introduction. (Fig 2A and B). 

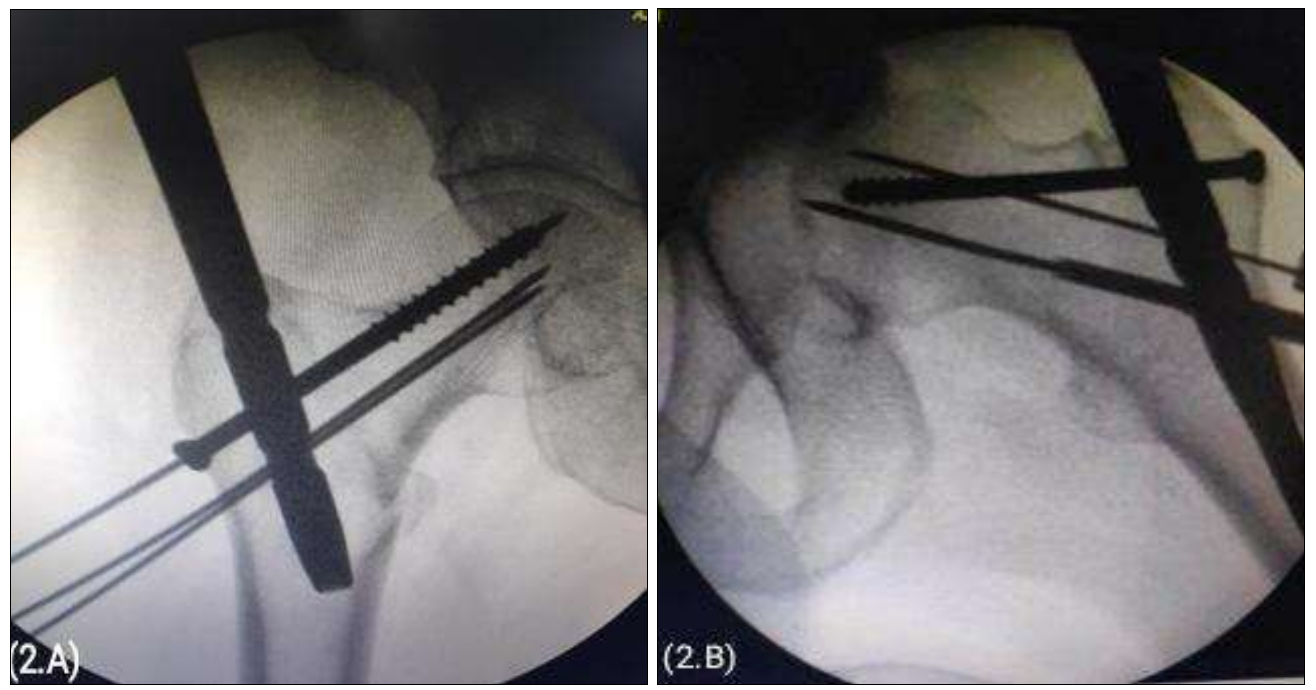

Fig 2A and B: (A) Shows the introduction of the IM nail into prefixed adducted limb with no opposition from eccentrically placed CC screw and (B) Further fixation with PFN screws to maintain reduction

$8 \mathrm{~mm}$ lag screw was placed through the nail into the neck under $\mathrm{C}$-arm guidance using the angled zig. Pre-fixation screw was removed, and regular $6 \mathrm{~mm}$ anti-rotation screw was placed through the zig. Alternate compression of lag and anti-rotation screw was done to achieve compression at fracture site. Distal locking of PFN completes the procedure followed by closure of fascia and skin. (Fig 3A and B).
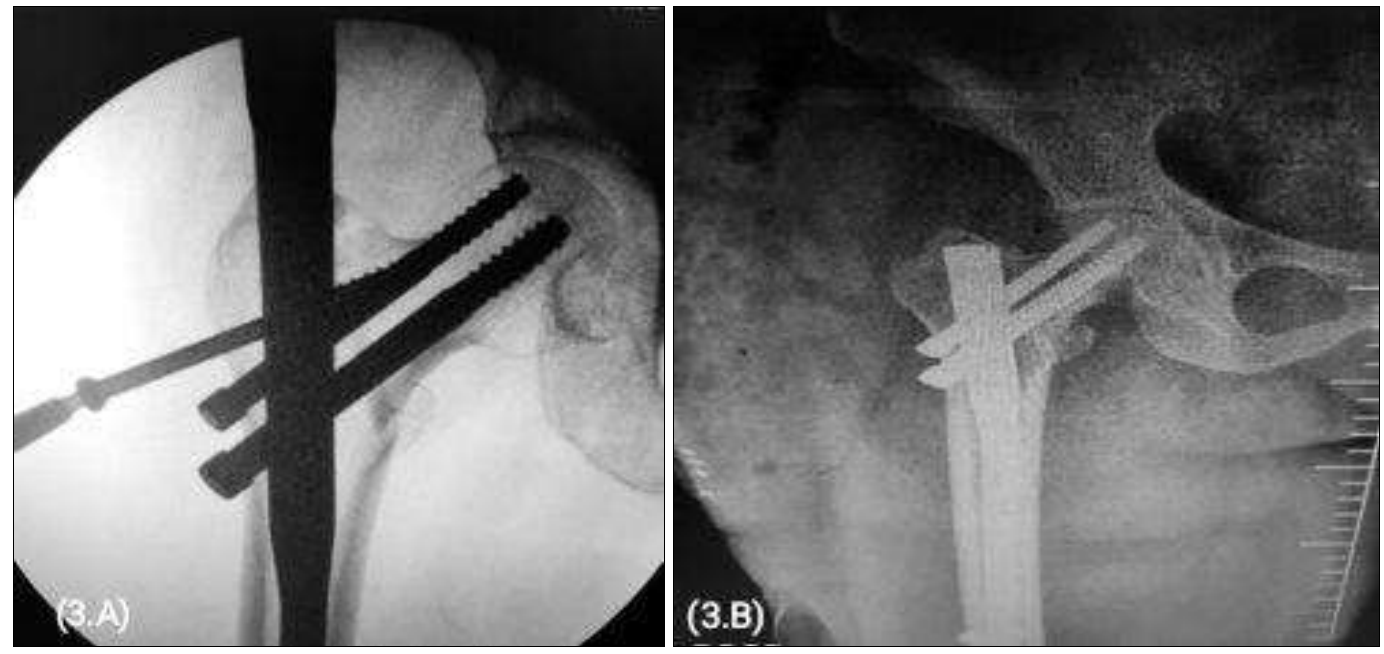

Fig 3A and B: (A) Shows withdrawal of pre-fixation screw and fixation of regular PFN fixation screws and (B) IT fracture post fixation with PFN with improved neck-shaft angle.

\section{Ethical consideration}

Ethical principles were followed while conducting the study. The protocol of the study was submitted to the ethical committee of the institution and permissions were also obtained from medical Superintendent of the medical college. Details of the patients were kept confidential.

\section{Statistical analysis}

Data was entered to Microsoft Excel (Windows 7; Version 2007) and analyses were done using the Statistical Package for Social Sciences (SPSS) for Windows software (version 22.0; SPSS Inc, Chicago). Descriptive statistics such as mean and standard deviation (SD) for continuous variables, frequencies and percentages were calculated for categorical variables were determined. Comparison of varus angle between pre-op, intra-op and post-intervention were analysed using paired-t test. Bar charts and pie charts were used for visual representation of the analysed data as shown in Graph 1,2 , and 3. Level of significance was set at 0.05 .

\section{Results}

In our series, majority of the cases i.e. 34 (73.9\%) belongs to age group of more than 60 years, followed by $12(26.1 \%)$ cases in the age group 51-60 years (range-54-81 years and mean age of 66.13 years). In our study, there was a female predominance [females-28 (73.9 \%), males-18 (26.1\%)]. Among 46 cases, 15 cases were belonging to $\mathrm{AO}$ classification $31 \mathrm{~A} 1.1,12$ cases were $31 \mathrm{~A} 1.2,15$ cases were 31A1.3 and 4 cases were 31A2.1. All the cases included in our study group were fresh fractures. All 46 patients underwent PFN and to prevent varus mal-reduction, above mentioned technique of pre-fixation screw was used in all the patients. During the surgery, mean blood loss among the subjects was found to be $34.67 \mathrm{ml}$ with average surgical time of 19.46 mins. Pre and post-surgery neck shaft angles were calculated and a significant improvement in the angles was noted. The mean neck shaft angle showed an improvement from 116.65 (pre-op) to 126.63 (intra-op) and then to 140.67degrees postoperatively. 


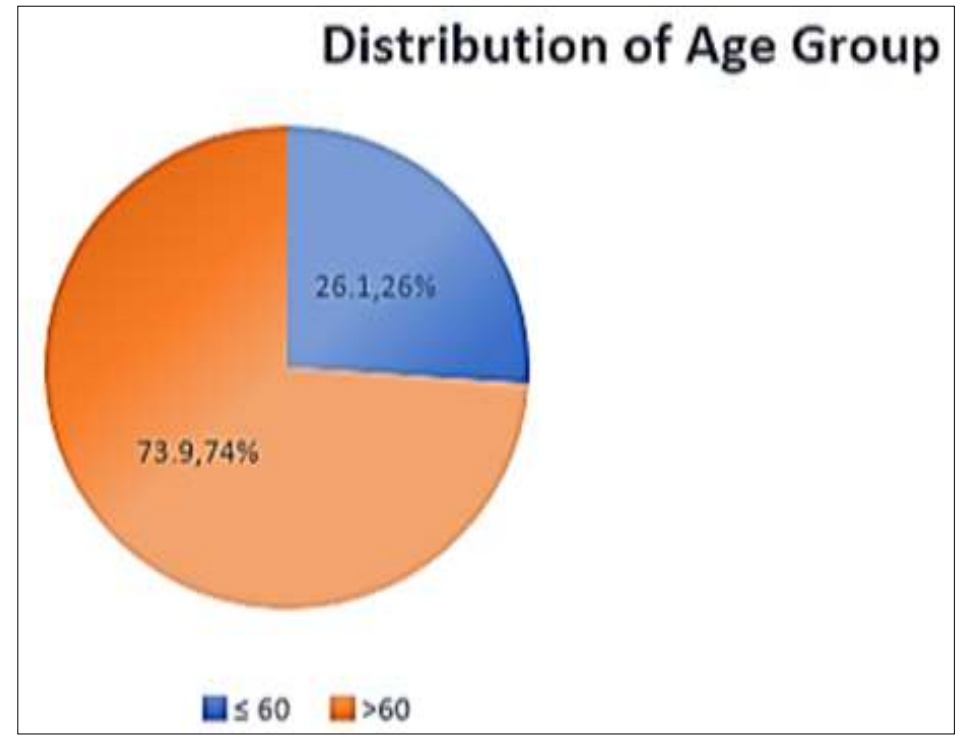

Graph 1: Schematic representation of age with maximum being above 60 years

Table 1: Distribution of study subjects according to the blood loss $(\mathrm{N}=46)$ showing decrease in average blood loss with above procedure

\begin{tabular}{|c|c|c|}
\hline Blood loss (In ml) & No. & Percent \\
\hline$\leq 30$ & 21 & 45.7 \\
\hline$>30$ & 25 & 54.3 \\
\hline Mean (SD) & \multicolumn{3}{|c|}{$34.67(7.91)$} \\
\hline Range & \multicolumn{2}{|c|}{$20-55$} \\
\hline
\end{tabular}

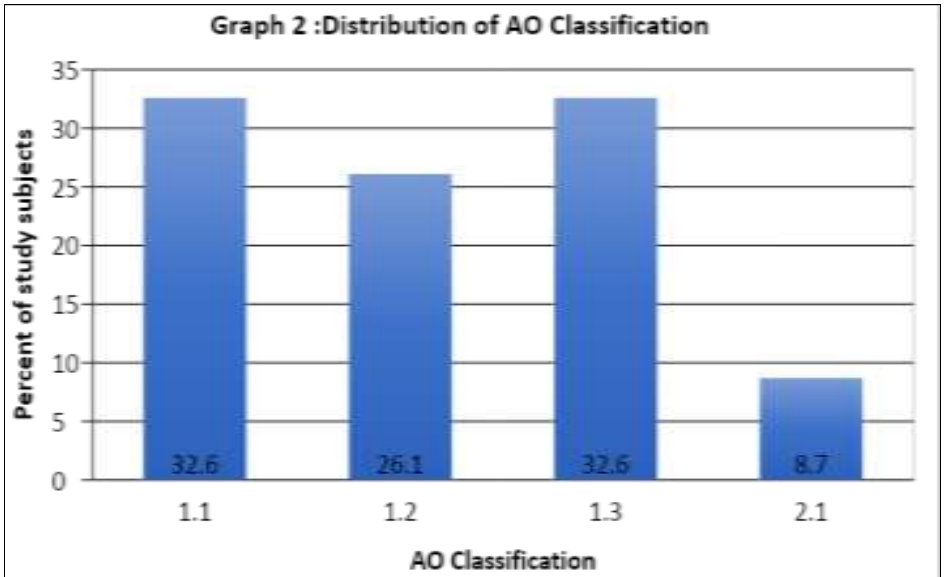

Graph 2: Schematic representation of classification of fractures involved in study with maximum of them falling under AO 31 A1.1 and 1.3

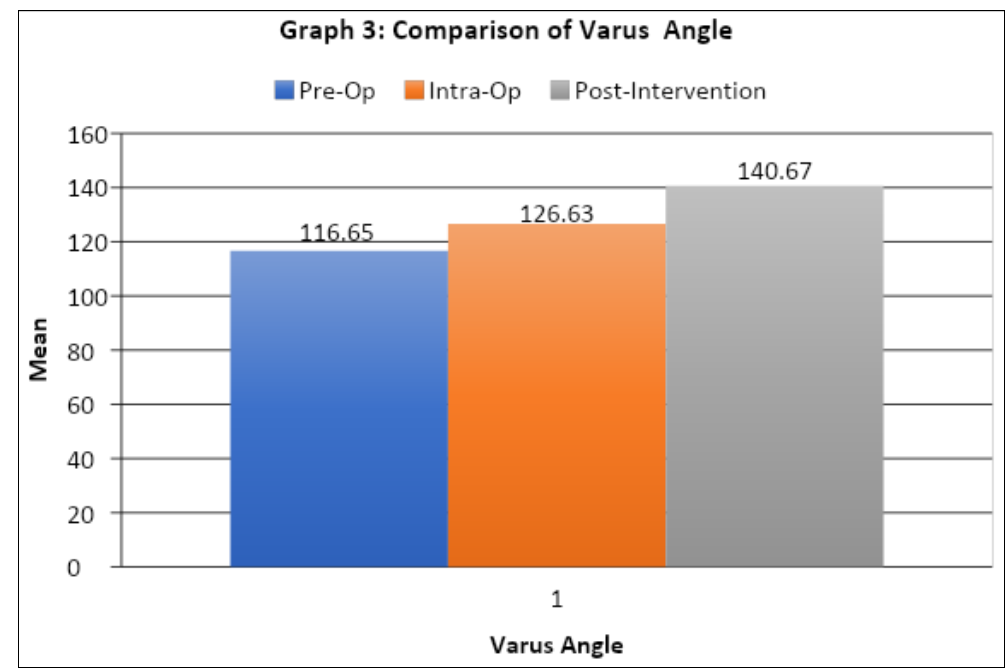

Graph 3: Schematic representation of comparison of varus angle pre-op, after reduction and post-intervention ( $\mathrm{N}=46)$ with significant improvement of varus angle of 14.04 degrees from reduction to post fixation 


\section{Discussion}

Dynamic hip screw has stood the test of time as an implant of choice for trochanteric fractures and more so for the stable patterns. Minimally invasive procedures have gained importance owing to the fact that these fractures occur in a fragile geriatric age group. Proximal femoral nailing has gained popularity as a procedure which can lessen the risk of morbidity and has a biomechanical advantage over DHS ${ }^{[8-10]}$. However, a good pre-reduction is the key for the successful outcome of PFN, hence in this study we evaluate the use of pre-fixation screw for maintaining valgus reduction ${ }^{[4,8-10]}$.

The maximum patients in our study were in the age group of 60-70 years with a mean age 66.13 years. In similar studies of comparison between PFN and DHS, PFN was the procedure of choice in this age group $[4,8-10]$. Jones et al., in his comparative study had a blood loss of $73 \mathrm{ml}$ for PFN and 159 $\mathrm{ml}$ for DHS ${ }^{[8]}$. Duration of procedure in his study was 59.50 min and $84.00 \mathrm{~min}$ for PFN and DHS respectively ${ }^{[8]}$. In contrast to this, our study showed significant reduction in duration of procedure (average $19.46 \mathrm{~min}$ ) as well as blood loss (average $34.67 \mathrm{ml}$ ). Selection of simpler fractures (AO classification) in our study could be the explanation for decreased duration of the procedure and reduction in blood loss. The change in the neck shaft angle in this study was significant with an average of 9.98-degree improvement from pre-op to intra-op where fracture reduction was obtained on fracture table. We further had an accent of valgus angle by 14.04 degrees after the application of pre-fixation screw, giving a final improvement of average 24.02 degrees. However, in a study done by Chun et al., where fixation of IT fractures using K-wires for maintaining valgus reduction was done, it did not show greater improvement in neck shaft angle (average 7.87 degrees), probably because of loss of reduction while passing the nail, which goes unnoticed.
Proximal femoral nailing is an effective treatment option for intertrochanteric fractures of the femur, bestowing the advantages of biological fixation and lessening risks of morbidity as well as complimenting with a biomechanically stable fixation ${ }^{[8-10]}$. Although this closed fixation system endows superior benefits, outcomes of PFN in intertrochanteric fractures rely mainly on the reduction achieved and maintained intra operatively ${ }^{[5]}$. There are innumerable technical hardships which can be encountered during the procedure making it difficult to achieve a good reduction and insert the implant in the desired position ${ }^{[13-14]}$. Few of the technical glitches are:

1. Achieving and maintaining reduction by correcting varus angulation, rotations, flexion of the proximal fragment and posterior sag of distal fragment.

2. Getting a desired entry point to insert the nail without disturbing the fracture reduction.

3. Placing parallel neck screws in appropriate position to maintain reduction and allow controlled compression at fracture site.

Proximal femoral nail is a closed nailing system with an external zig meant for fixed angle screws and is designed for an anatomically reduced fracture. K Semmi, et al. reported that the quality of fracture reduction is an important factor that affects the revision rates and mechanical failures after osteosynthesis with PFN for trochanteric fractures ${ }^{[15]}$. Hence pre-reduction is of paramount importance to be able to place the neck screws in desired position. Since we are dealing with fracture morphology where the fracture site is in the vicinity or sometimes at the nail entry site, it is observed that it disturbs the fracture reduction while inserting the nail. Varus mal-reduction (figure Fig $4 \mathrm{~A}$ and $\mathrm{B}$ and 5) is a consequence of this technical glitch ${ }^{[14]}$. (Fig 4A and B) (Fig 5).

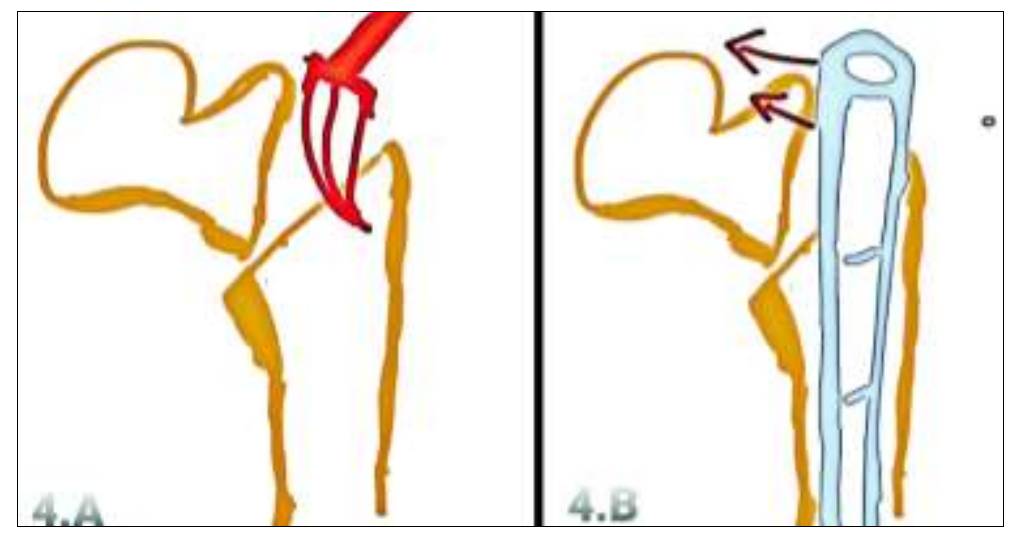

Fig 4A and B: Image (A) illustrates the opening up fracture segment with awl and image (B) illustrates subsequent nail insertion

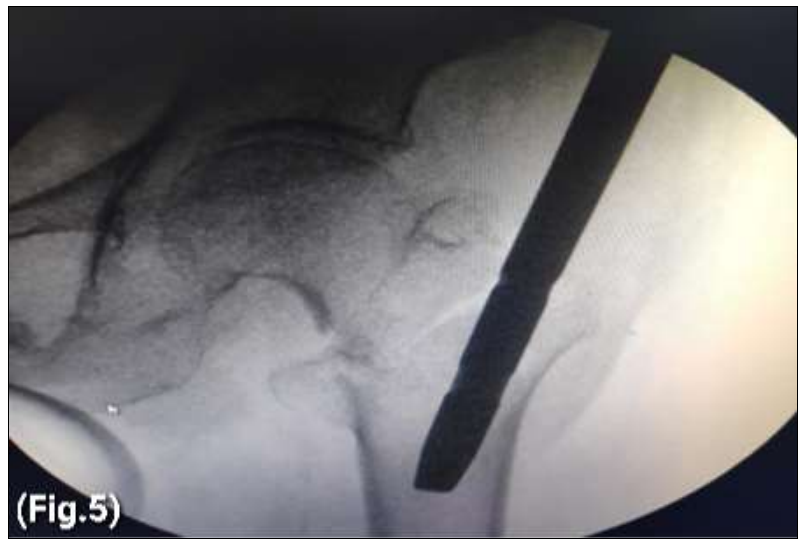

Fig 5: Proximal femoral segment going for varus mal-alignment with PFN nail placement. Nail entry through fracture site act as a
Making a trochanteric entry with a bone AWL and inserting the nail through the tip of the trochanter is convenient in adducted position of the hip when the affected limb is placed in fracture table ${ }^{[16]}$. When there are no fractures in and around the greater trochanter, adduction of hip would align the trochanter and femur shaft away from the pelvis facilitating the procedure of nail entry. However, in trochanteric fractures, it is observed that reduction achieved in neutral or abducted position of the hip cannot be maintained when the hip is forced into adduction. Such kind of fracture patterns especially AO 31 type A 1 are suitable for DHS which can be conveniently done in abducted position of the hip. Hence varus mal-reduction (figure 6 and 10) occurring due to nail entry position is yet another technical glitch. (Fig $6)$. 


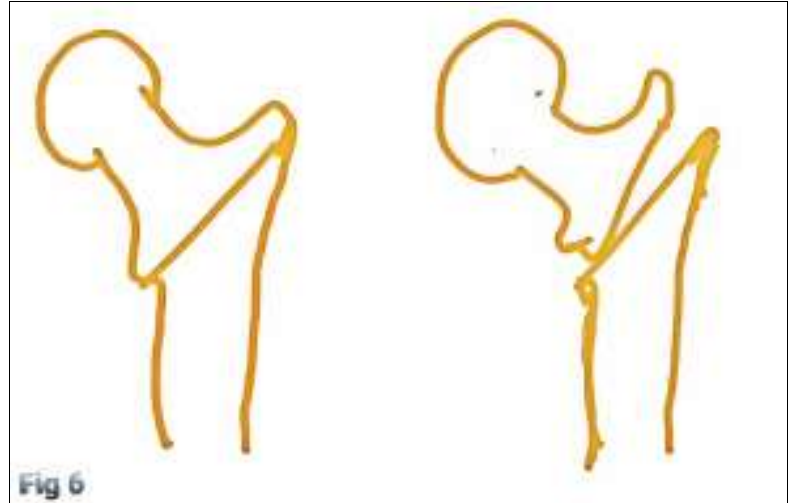

Fig 6: Schematic representation showing proximal segment of proximal femur reduced in abduction on fracture table which goes to varus as soon as limb is drawn to adduction

Correction of varus achieved by either using schanz pin in the proximal fragment or by using bone hook into the pyriform fossa has been explained in literature ${ }^{[17]}$. Neck shaft angle can be altered appropriately by positioning of the hip in adduction or abduction. Pre fixation with K-wires to maintain valgus reduction while hip is positioned in adduction has been one of the technical tips, although there are a few impediments observed with the use of K-wires. Firstly, K-wires do not get good enough purchase from the bone for maintaining reduction in an osteoporotic bone. Secondly, the pull of stronger abductors in relatively younger age group can bend the k-wires with consequent loss of valgus reduction. Lastly, $\mathrm{K}$-wires in situ which will be projecting out of the skin can increase the inconvenience of inserting the nail with the jig components and also placing drill sleeves necessary for passing the neck screws.

\section{Advantages of having a pre-fixation screw for maintaining varus reduction}

Partially threaded cannulated cancellous screws can be placed

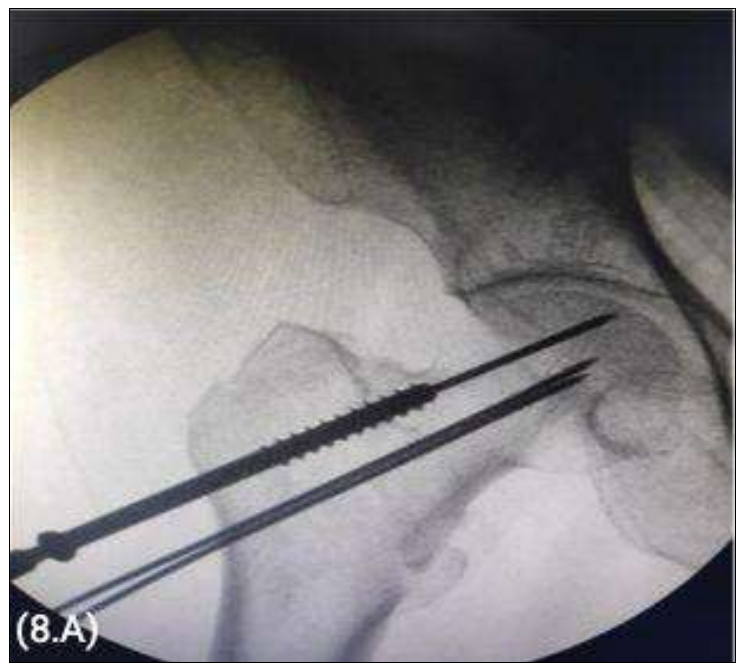

Fig 8A and B: (A) Pre-fixation of IT fracture with cc screw and (B) Maintaining reduction with nail passage

\section{Drawbacks}

1. Screw is a relatively thicker device when compared to Kwires and hence there is a possible fear of difficulty in inserting the nail. This can be overcome by precisely placing the screw parallel and close to anterior cortex of femur using guide wire under fluoroscopic guidance. We have observed that there is more than enough space to place the nail centrally in the trochanteric area even with

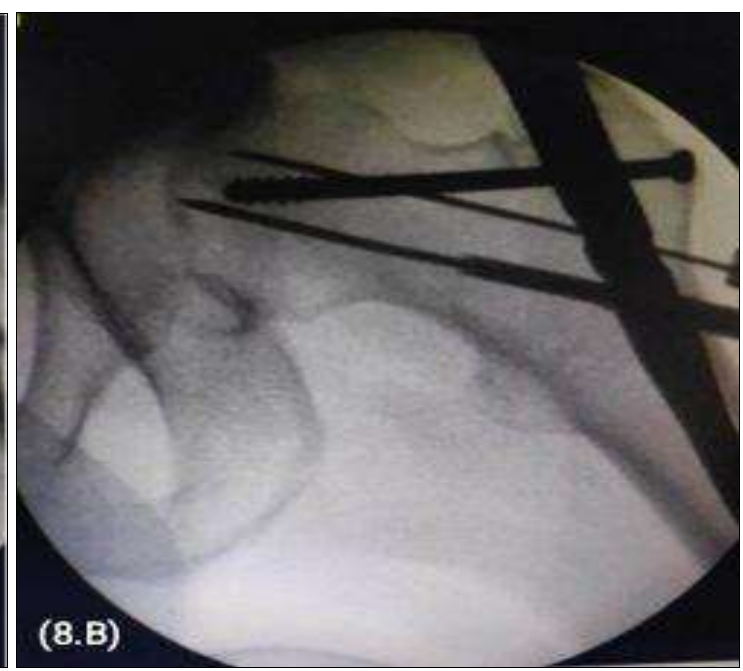

eccentrically on the proximal half of the fracture line using a guide wire under fluoroscopy guidance. This screw provides compression at the proximal half of fracture line, creating a subtle correction of varus and maintains the valgus reduction when limb is positioned in adduction. In contrast to K-wires, screw has an advantage of providing compression in the fracture line due to the lag effect. This locks the fracture fragments in compression mode and hence, reduction is maintained throughout the procedure. Screw is a thicker and sturdier device which does not bend on forceful adduction even in a relatively young bone. Moreover, cancellous threads have an advantage of getting a better purchase in the head of the femur of osteoporotic bones and thereby preventing any loss of reduction and displacement into varus which occurs more frequently during the insertion of nail through fracture site. This is easily avoided because the fracture fragments are in compression mode and at the same time prevents the nail to act as a spacer in between fracture fragments as shown in figure 7 and figure $8 \mathrm{~A}$ and B. (Fig 7) (Fig 8A and B)

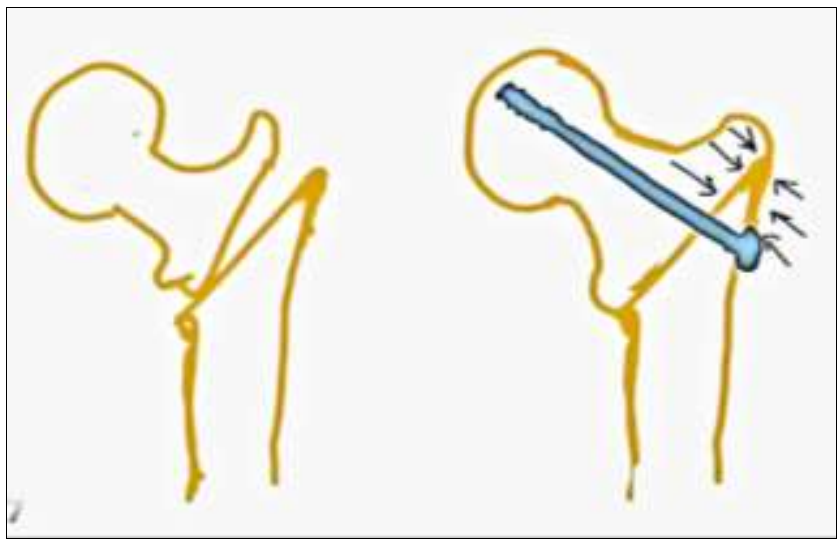

Fig 7: Schematic representation showing varus mal-alignment (left) and its reduction with pre-fixation compression screw giving an improvement in varus angle

the pre-fixation screw in situ.

2. Eccentric compression of screw can sometimes force the fracture into valgus mal-reduction which can be avoided by controlled compression under fluoroscopic guidance. (Fig 9). 


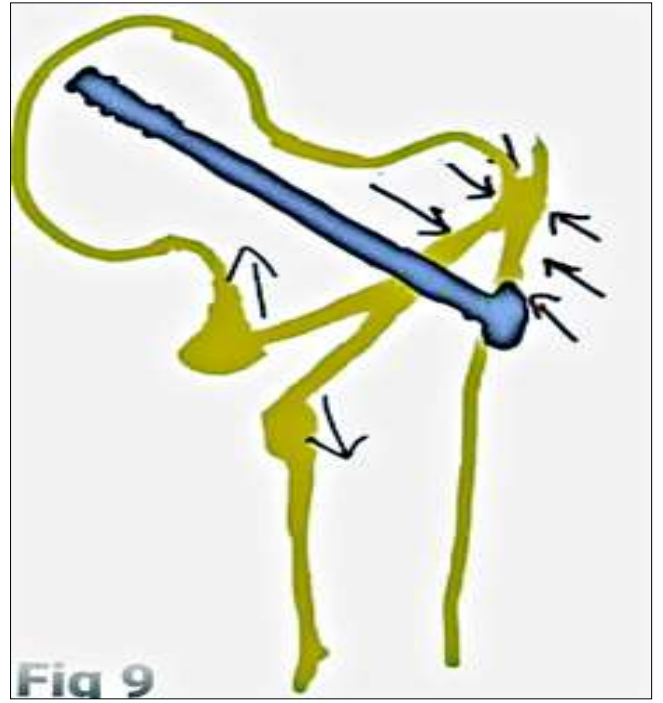

Fig 9: Valgus mal-reduction with eccentric pre fixation screw

1. Screw cannot be used for reduction when there is comminution in the lateral cortex, in such instances screw would sink inside the lateral cortex and may not serve the purpose.

2. This technique doesn't work for reverse oblique or subtrochanteric fracture patterns.

3. Correction of flexion in proximal fragment, mal-rotations and posterior sag of trochanter cannot be achieved by this technique and needs independent manoeuvres.

In patients with good bone quality and younger age group, we observed that compression screw aided in better reduction and it was necessary to ream the canal prior to nail entry. It was also noticeable that pre-fixation screw had no hindrance for the passage of guide wire and the reamers. In our study we had 4 patients with fractures belonging to AO 2.1 group. In these fractures we were able to achieve reduction by prefixation screw technique since there was minimum comminution in the trochanter. Mild posterior sag was observed in the lateral view, which was corrected by minimal pressure on the posterior aspect of trochanter prior to passing the guide wire for pre-fixation screw. However, this manoeuvre did not increase the complexity of the procedure and hence were included in the study, unlike fractures with extensive comminution (A0 31 A 2.2, 2.3).

\section{Conclusion}

Minimally invasive procedures should be preferred for fractures occurring in geriatric age group. PFN is relatively less invasive procedure when compared to DHS in Simple intertrochanteric fractures occurring in elderly due to trivial fall. Maintaining reduction throughout the procedure is a challenge in PFN owing to certain technical aspects associated with the procedure. Pre-fixation screw emerges as a good technique in maintaining reduction throughout the procedure and thereby reducing varus mal-reduction. However further Randomize control studies with adequate sample size and longer follow up in this aspect has to be undertaken to draw conclusive evidence.

\section{References}

1. Koval KJ, Chen AL, Aharanoff GB, Egol KA, Zuckerman JD. Clinical pathway for hip fractures in the elderly: the Hospital for Joint Diseases experience. Clin Orthop Relat Res 2004;425:72-81.
2. Voleti PB, Liu SY, Baldwin KD, Mehta S, Donegan DJ. Intertrochanteric Femur Fracture Stability: A Surrogate for General Health in Elderly Patients?. Geriatric orthopaedic surgery \& rehabilitation 2015;6(3):192-6.

3. Pathania VP, Sharma M, Gupta S, Kaushik SK. Management of intertrochanteric fracture by PFN Vs DHS: a comparative study. Journal of Evolution of Medical and Dental Sciences 2015;4(39):6741-51.

4. Rajarajan NS. A comparative study of treatment of unstable intertrochanteric fractures with PFN and cemented hemiarthroplasty. International Journal of Orthopaedics 2018;4(2):111-5.

5. Marmor M, Liddle K, Buckley J, Matityahu A. Effect of varus and valgus alignment on implant loading after proximal femur fracture fixation. European Journal of Orthopaedic Surgery \& Traumatology 2016;26(4):37983.

6. Chang WS, Zuckerman JD, Kummer FJ, Frankel VH. Biomechanical evaluation of anatomic reduction versus medial displacement osteotomy in unstable intertrochanteric fractures. Clin Orthop Relat Res 1987;225:141-6.

7. Desjardins AL, Roy A, Paiement G et al. Unstable intertrochanteric fracture of the femur: a prospective randomised study comparing anatomical reduction and medial displacement osteotomy. J Bone Joint Surg Br 1993;75(3):445-7.

8. Jonnes C, Shishir SM, Najimudeen S. Type II intertrochanteric fractures: proximal femoral nailing (PFN) versus dynamic hip screw (DHS). Archives of Bone and Joint Surgery 2016;4(1):23.

9. Bakshi DA, Kumar DP, Brar DB. Comparative study between DHS and PFN in intertrochanteric fractures of femur. IJOS 2018;4(1):259-62.

10. Kyavater BS, Gupta S. Comparative study between dynamic hip screw vs Proximal femoral nailing in unstable inter-trochanteric fractures of the Femur in adults. Journal of evolution of medical and dental sciences-jemds 2015;4(50):8690-3.

11. Chun YS, Oh H, Cho YJ, Rhyu KH. Technique and early results of percutaneous reduction of sagittally unstable intertrochateric fractures. Clinics in orthopedic surgery 2011;3(3):217-24.

12. Lourenço PR, Pires RE. Subtrochanteric fractures of the femur: update. Revista Brasileira de Ortopedia 2016;51(3):246-53.

13. Kulkarni GS, Limaye R, Kulkarni M, Kulkarni S. Intertrochanteric fractures. Indian journal of Orthopaedics 2006;401:16.

14. Siddiqui YS, Khan AQ, Asif N et al. Modes of failure of proximal femoral nail (PFN) in unstable trochanteric fractures. MOJ Orthop Rheumatol 2019;11(1):7-16. DOI: 10.15406/mojor.2019.11.00460

15. K Şemmi, A Taşkın, K Cemil et al. Mechanical failures after fixation with proximal femoral nail and risk factors. Clin Interv Aging 2015;10:1959-1965.

16. Tank PJ, Solanki RA, Patet HK, Rathi NV, Misttry J, Bhabhor HB. Results of proximal femoral nail in intertrochanteric fracture femur. Int J Med Sci 2016;1:1724.

17. Gadegone WM, Shivashankar B, Lokhande V, Salphale Y. Augmentation of proximal femoral nail in unstable trochanteric fractures. SICOT-J 2017;3. 